\title{
Research on Blended Teaching Model of College English Courses from Perspective of Flow Theory
}

\author{
Qixin Liu \\ Faculty of Foreign Languages \\ Huaiyin Institute of Technology \\ Huai'an, China
}

\begin{abstract}
Flow Theory has been widely and successfully applied in realms like positive psychology, game designing, product promotion, etc. Flow, as the name implies, refers to an optimal experience people may go through when they are undertaking tasks that are challenging yet intriguing enough to evoke their maximum dedication to the tasks. In the process, people forget about time and themselves, and pay no attention to the change of the external environment. Blended Teaching Model, advocating the integration of classroom instruction and online learning, has emerged as one of many prevalent means educators and teacher implement to enhance college English teaching quality. This paper tries to explore the application of Blended Teaching Model to college English courses from the perspective of Flow Theory which sheds light on redesigning class and evaluating the learning effect.
\end{abstract}

Keywords-flow theory; college English course; blended teaching mode; course design

\section{INTRODUCTION}

As the concept of "Internet Plus" has merged into immense areas and fields, education is no exception, among which, higher education, with its heightened mission and pioneering function, plays a leading role in implementing this concept into classroom instruction practice. College English course, as one of core and fundamental courses in university, has been the hot land of research for educators and the literate, showcasing vibrancy endowed by the advent of the Internet. In recent decades college English course has witnessed the prevalence of persistent and bold exploration of researchers and teachers on means to adjust the instruction modes to the ever-changing and diverse needs of students, who admittedly, under tremendous influence of the Internet more than any other elder generation. With more than one mode of instruction adopted into classroom: Flipped Class, Project-Based Learning(PBL), MOOC, etc., the conclusion can be easily drawn that the Internet has already penetrated into and facilitate the teaching process of college English on various levels, eliminating the traditional barriers that hinder students' learning. However, like each and every newlycreated invention, lack of systematic and chronic research and exploration may as a result lead to unclear perception and unreasonable expectation. According to a variety of research, combination of college English courses and application of the Internet have exerted seemingly satisfactory results, which, however, seems unqualified and inadequate for responsible researchers who quench for the answers to puzzling questions like how should instructors design the teaching process and how to evaluate it? It would make no sense if the outcome still be decidedly relevant to students' grades rather than how they feel about their performance in the learning process. And how can students be intrinsically motivated to get engaged in a teaching activity?

"Flow Theory", which sets forth "optimal experience", proposed by Csikszentmihalyi, might be the answer to the questions above and according to him, it is a "peculiar dynamic state-the holistic sensation that people feel when act with total involvement" andan "ordered, negentropic state of consciousness", which implies the happiness and sense of achievement one can obtain from total dedication into one's obligation [1][2]. When experiencing flow, people may not notice irrelevant outer stimuli and fixating their total attention on the task at hand. As adults, college students face various disturbance and distraction from both outer and inner world, and the intense entertainment brought by the Internet and mobile phone make it even harder to resist the temptation, which leads to the more complicated issue of class monitoring. Therefore, it is reasonable to extrapolate that the likelihood of problems discussed above in Blended Method Teaching getting solved will increase with the Flow Theory incorporated.

\section{FLOW THEORY AND BLENDED TEACHING MODEL}

\section{A. Definition of Flow Theory}

Csikszentmihalyi first came up with the idea of "flow" in the book Beyond Boredom and Anxiety after he observed the working procedure of people from different walks of life. By observation, he observed that the people interviewed, though from a spectrum of professions, genders, cultures, consented to one related state where they were whole-heartedly drenched into the ongoing activity with time passing unnoticed and attain the enjoyment arising from it. It refers to a mental state where people commit 100 percent attention and endeavors to accomplish the task without being distracted while feeling rewarded and delightful when going through it. Afterwards considerable attempts have been expended to conduct research and experiment around Flow Theory, becoming the benchmark of the Positive Psychology 
and also applied to various realms and subjects. Through all these researches, it is uncovered that people get more productive and innovative when they are in the state of flow and some criteria are proposed to be met to reach optimal experience.

\section{B. Variables of Flow}

Just like when people sometimes describe themselves as "being in the zone", flow is such a mental state that people may experience in various activities, such as playing online games, performing arts, doing sports, learning new stuff, working, etc. To get fully exposed to flow, certain criteria need to be met or to put it another way, some universal characteristics comprise the essence of flow: challenge and skill, a clear objective, feedback, concentration, mergence, sense of control, time distortion, loss of self-consciousness and enjoyment.

- Challenge and skill. To explain on what ground can people sense the flow, scientific and psychological experiments, where the subjects were asked to note down what they were doing and how they felt at the moment every a few hours, have been conducted to obtain the data facilitating researchers' perceiving of the pre-conditions and the traits of flow. The balance between one's skill and challenge is one of the prerequisites of flow that can be explained on two levels. On the one hand, the activity must be associated with skills, or in other words, certain skills are in demand for achievement of the task. In other words, flow will not occur to the person if there is absence of skills in the task [3]. People can reach the flow state on the condition that they master perceived skills that are relatively high and the challenge encountered in the task is also relatively high. If the challenge of task is high while the possessed skills are relatively lower, the person may feel anxious rather than a sense of conquering and fulfillment; if the challenge of the task is relatively low while the person is very skillful, the person may comprehend such a sense of boredom that he or she gradually loses interest in pursuit of terminating the task.

- A clear goal. Goals are divided into short-term goals and long-term goals, providing people with a sense of purpose and also get people prepared on mind level for the upcoming task. The goals, no matter shortterm or long-term, should be clearly-structured so that people can have an intuitive sense of their progress along the upward ladder.

- Feedback. It indicates that "quick and ambiguous feedback" must be conveyed to the task performers so they can receive a quick response of the success of the task at hand. Basically people need to have an initial feeling of what kind of outcome comes from their performance.

- Concentration. In the task, the task is designed elaborately to be intriguing to the task performers so that they may reach a heightened state of concentration and not prone to be distracted by outer change.

- Mergence. When experiencing flow, people's ego will fade and they are unconscious of their presence in the process;

- Sense of control. During the activity, the task performers feel capable of executing control over the whole process and generate a reasonable anticipation about the outcome.

- Time distortion. When experiencing flow, people may lose the normal sense and perception of time as time may be perceived as accelerating or decelerating.

- Loss of self-consciousness. When implementing the task, people involved may not only lose track of time, but also be incapable of sensing the existence of themselves.

- Enjoyment. The task proves to be intriguing and appealing to the task performer; besides, the involvement is self-rewarding thus needing no outer stimulus. Therefore, the more people get involved into the activity, the more they want to prolong it. Happiness and productivity as well will hence be fueled.

\section{Implication of Flow Theory in College English Courses}

College English courses, due to its large-scale student numbers and limited location and time of class, are encountered with impediment with respect to teaching mode reform. Compared to primary school and high school instruction that burden students with monotonous in-class lecturing and after-curriculum drill, college provides a broader and freer stage for teachers and students to make class alive with incorporation of various teaching methods. After numerous have been tried in transforming college English teaching, there has been lack of unified ruler to evaluate its success, which Flow Theory, if implemented scientifically and thoroughly, can be of great value and influence to the teaching field. In teaching, the eight characteristics of flow can be summarized into four aspects in terms of measuring whether student participants can reach the flow state.

- Whether the learning goal is clear and the participants are strongly motivated to accomplish the task.

- Whether the balance between skills and challenge has been met so the participants are confident, competitive and eager to accomplish the task.

- Whether enough interaction and cooperation between participants are in demand in the task so they can better experience flow and heighten their sense of achievement through learning from cooperation.

- Whether other factors have been taken into consideration like each individual's cognitive ability, attitude and emotion, etc. 


\section{Blended Teaching Model}

In the year of 2017, the 13th Five-Year Plan for Economic and Social Development of the PRC (the 13th Five-Year Plan) issued by the State Council announced that information technology and education industry should be merged deeply and various methods of teaching should be adopted to forge into a new mode combining online and offline learning, which composes a blueprint for the reform of college English to incorporate online and offline resources for intensified teaching effect. This document provides a broader area for English course teachers in terms of utilizing this opportunity to transform the traditional in-class teaching in order to ameliorate lifeless teaching. Blended teaching model focused on integrating students' constructive learning with assistance of modern technology to facilitate deep learning. Students online can explore different methods and utilize individualized learning instruments to meet personalized learning goals, which infuses boosted pleasure and challenge into the task for students. The online activities can produce simulated real-life situation where abstract knowledge is made concrete and comprehensible so the task is of tremendous appeal to challengers, which can be addressed by them with proper skills under supervision. While triumphing the task, people can generate a sense of fulfillment and delight.

Focusing on this point, accumulating interests are gathered on courses redesigning while comparatively less attention are shifted to effect evaluation of students, who supposedly are the center of the blended teaching method. Furthermore, while instilling new blood into teaching, blended teaching faces its newly-born issue: loose monitoring, easily distracted attention, low self-motivated participation, short of practice opportunity, etc. Therefore, it is of gigantic significance to explore college English learning from perspective of Flow Theory taking into account the effect they have on students' learning.

\section{TEACHING PROCESS DESIGN}

The teaching process designed by the author based on the Flow Theory can be shown in "Fig. 1".

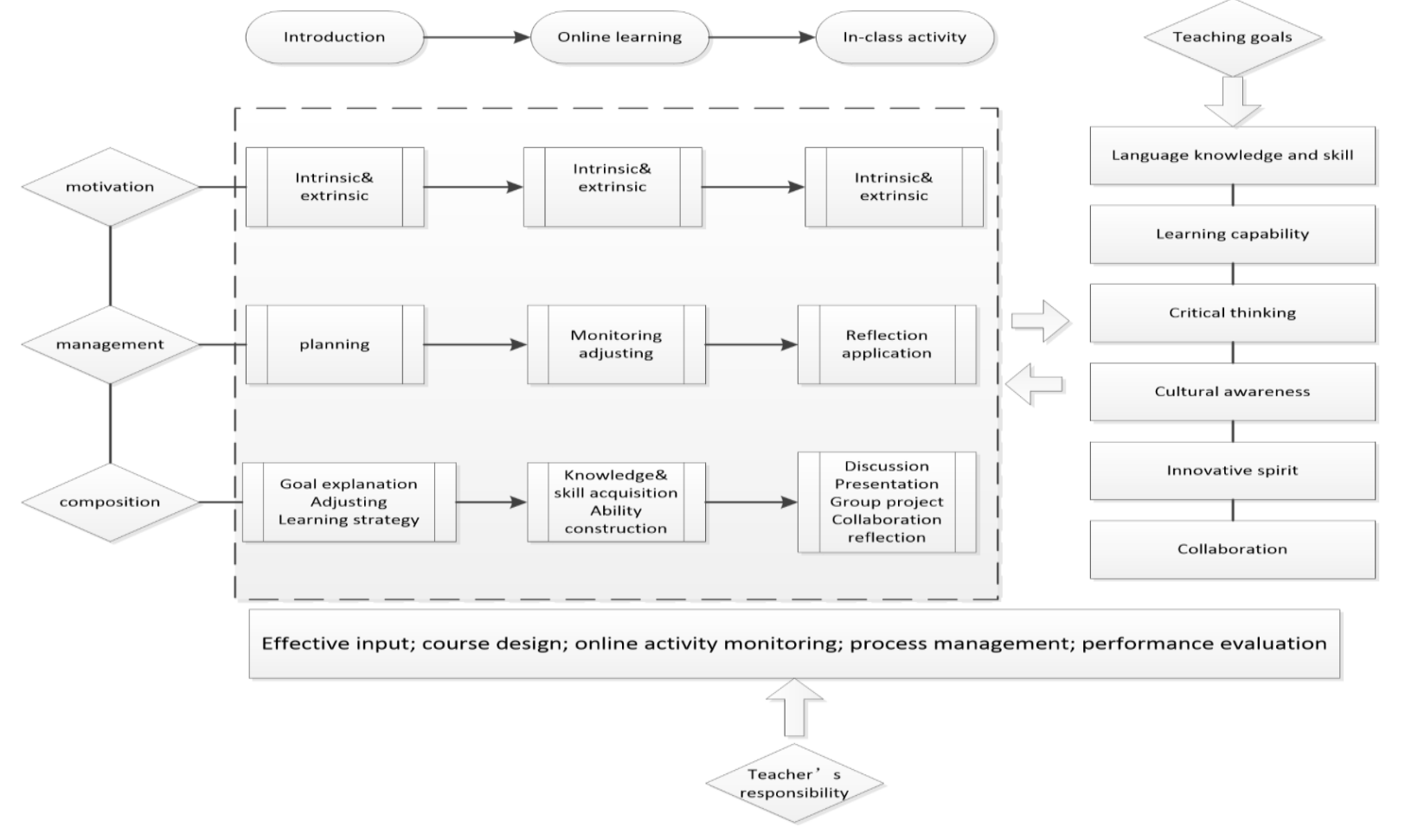

Fig. 1. Class design based on Flow Theory.

People take certain actions based on two kinds of motivation: intrinsic and extrinsic. When getting involved in blended teaching model, both types are also required. When students are intrinsically motivated, they are unaware of themselves as well as time and they only fixate their attention on performing the task. Time and space means nothing to them and all that matter is the mission to be accomplished. For example, when painters draw a picture, they only focus on how to best capture or express the view in front. Likewise, when students are internally driven, they will forget ego, go beyond themselves and quench for more knowledge and skills to conquer more abstruse mission. That is where a flow can emerge and sustain the learners to go through the whole procedure. Extrinsic motivation signifies outer reward, which not necessarily means tangible payment or prize, but also represents intangible approval or encouragement from peers or the authority like teachers. Accordingly, if extrinsically motivated, students will not necessarily look for outer rewarding, but instead will be itching for teacher's approval and peers 'encouragement, which are all demonstration of extrinsic reward. Therefore, it 
is of great necessity for teachers to insert peer evaluation and instant feedback from the teacher into designing class.

The teaching goals concerning English courses fall into six categories: language ability, learning capability, critical thinking, cultural awareness, innovative spirit and collaboration. Language ability are the most basic involving students' command of knowledge and skill, for example, when will third person singular be used? Learning capability refers to the ability to learn a language, such as being capable of inferring the meaning of a new word based on context without looking it up in the dictionary or pronouncing a new word based on the spelling pattern and rule. Critical thinking, core ability according to some course syllables, proposes that students are not containers like glasses waiting to be filled by teacher with knowledge. Combining specialized college courses with training of critical thinking can strengthen students' critical thinking and enhance the study of their major expertise as well [4]. The reason for students to get information, knowledge and skills is to distinguish, to reason, to criticize and to appreciate. Cultural awareness is the ability students need to have to appreciate certain culture and compare different cultures. Innovative spirit is a capability to contemplate things from a novel perspective and solve problems in a creative way. It is of great necessity for college students to cultivate innovative skills to develop into allround graduates. Cooperative skills are emphasized for quite some time due to high demand for cooperation in workplace nowadays to conquer more complicated tasks with higher risk and greater challenge. Staffs with adequate communicative and cooperative skills working with synergy manifest more competence compared to lonely wolves. The experience of flow of learners during the class will exert a positive influence on them acquiring those skills. It can strengthen students' cognitive ability, enhancing their logical reasoning and deducing competence when tackling simulated situations. Moreover, loss of their ego and time will make students more comfortable and at ease to embrace new knowledge and more open to cooperation with peers, which fuels their innovation, cooperation.

Management expertise and management skills are required for an effective class. It is the same with online blended mixed teaching. Before the online class, in the introduction class, students need to be assisted by instructors with formulating a plan at first based on the pre-assessment evaluating their language competency and skill level. When laying down the plan, a clear goal should be set like what they will achieve in a specific class in terms of knowledge, skill and awareness. Besides, the teacher also needs to make students aware of how to use the software related if necessary. Afterwards taking the online course is a process of students' self-monitoring and self-adjusting. Students can monitor the course pace, their state of mind, and control other outer factors that may exert influence on learning. Furthermore, during the learning process, students need to keep adjusting themselves to the ongoing activities: "what learning strategy am I going to use to tackle this problem?" or "What is the video trying to convey?" In the classroom instruction part, students need time to digest and consolidate the content they have acquired in online part. The teacher can organize students in form of reflection, summarizing, group discussion, presentation, application, solving problems, etc. Throughout learning blended with the Internet assistance and in-class instruction, students are able to manage themselves to maximize the effect of learning. A strong and effective management of the class can better facilitate the occurring of flow.

Admittedly computers have become one key element in transforming the traditional classroom teaching, but the truth is that teacher's role is much elevated than ever before instead of being ignored. In Blended learning, teachers' responsibilities are manifested on five levels: effective input, human-computer interaction, class design, process management, post-assessment evaluation. Effective input refers to the instructions given by the teacher on language acquisition and the demonstration of certain learning skills which will be delivered to the students in introduction class before the online course. In human-computer interaction part, first of all, elaborate instruction should be given on how to use the software or where can users find the proper resources -tips on how to adjust students to new way of learning. The online learning can take various forms like playing games specially designed to achieve certain educational goals where students learn stuff through passing each part or watching teaching videos about the certain content, which should be carefully and scientifically designed or chosen by the teacher. If the blended learning part is designed delicately to achieve the balance of skills and challenge, the students might find the interaction with computer intriguing and yearn for tasks more complicated and more challenging, which to some extent poses difficulties for teachers-because online teaching part may no longer be just watching teachers lecturing on the Internet and doing exercise afterwards. The kind of its forms can go beyond people's imagination and teachers should not stop exploration. Online learning part also requires the teacher to accompany students monitoring their learning, assisting them with proper learning strategies and necessary knowledge and check on them to avoid occasional outer distraction. Thus the likelihood of students reaching a flow state is much increased and satisfactory. The online teaching is followed by the in-class coaching where the teacher undertakes the role of leading students to summarize the content they have learned, reflect which part are still confusing and give feedback instantly. Moreover, the teacher should present an overall comment or evaluation on students' behavior throughout the whole process. Interestingly, this part, if reconstructed properly, can also trigger a flow in students. For example, in a class that is themed "play", students can form a group and prepare to present a play to the whole class or give a presentation introducing some of the best plays in the world. The rest of the students can give each group marks based on their performance. After adequate preparation with proper instruction, students are capable of enjoying themselves by giving a remarkable presentation or performance and leaving a good impression on their peers. To encourage participatory learning among students that can fuel students to reach flow, teachers need to bear in mind a couple of key points: to match the challenge of class and skills of students, to demonstrate a clear-structured goal, to facilitate cooperation 
among students, etc. In a nutshell, only with the management in class can flow be realized and it in turn shapes the various aspects of the learning outcome.

\section{CONCLUSION}

College English teaching has been a hot spot topic for research for decades due to its significance of equipping students with not only language proficiency but other skills like critical thinking, metacognitive capability, collaboration, innovation and so on. However, in front of college English teaching, there is still a long way to go in helping students accomplish those objectives. Multiple purposes and various demand of students, loose classroom management, lack of motivation and teaching method, ineffective assessmentthese are all common impediments to be addressed in college English teaching. Blended teaching mode may provide a popular and feasible way, yet it is not a panacea. It only works under right direction and on solid theoretical foundation. And in language acquisition, Effective Input theory submits that it is the most optimal situation when difficulty of learning materials are just one level above the learners' capability so that the material can neither be too demanding nor plain for them, which calls for elaborate design of the course outline, course plan and course content as well. What is fascinating is that it is consistent with the Flow Theory suggesting that it is only when the capability of people and challenge of tasks are compatible with each other that people will experience flow, feel intrinsically driven to pursue more complicated tasks and keep pushing their boundaries. Therefore In this paper, a searching proposal is posed that maybe the real problems in blended college English teaching can be tackled from the perspective of Flow Theory. In language learning, students will not fathom difficult tasks as something unconquerable; instead, they will try each means to work it out like going on the Internet or asking the teacher for help rather than quit until it is done. It is in process like this that students can make huge breakthrough in mastering not just language points or vocabulary usage, but also learning skills, self-management and putting something theoretical into practical use. It is based on the Flow Theory that the author proposes a new mode of class designing.

One restriction of this paper is that the whole lesson is only divided by the author into three parts: introduction, online learning and the in-class coaching, which is not a thorough arrangement of each part. A real class with blended teaching should include pre-assessment, introduction, reading materials, online learning, discussion, assignment, assessments, creation and practice, each and every of which requires further detailed discussion and exploration.

\section{REFERENCES}

[1] Mihaly Csikszentmihalyi, Beyond Boredom and Anxiety. San Francisco: K. Jossey-Bass Publishers, 1975, pp.36.

[2] Mihaly Csikszentmihalyi and Isabella Selega Csikszentmihalyi, Optimal Experience: Psychological Studies of Flow in Consciousness. Cambridge: Cambridge University Press, 1988, pp.34.
[3] Keller, J., and Landhauber, A., "The flow model revisited," in E. Stefan (ed.), Advances in Flow Research. New York: Springer, 2012, pp 51-63

[4] Hitchcock D., "Critical thinking as an educational ideal," Journal of Higher Education. Wuhan, Vol.33, pp.60, Nov 2012. 